\title{
Relationship between local and systemic inflammatory response and prognosis in laryngeal squamous cell carcinoma
}

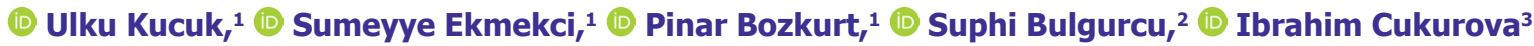 \\ ${ }^{1}$ Department of Pathology, Tepecik Training and Research Hospital, Izmir, Turkey \\ 2Department of Otorhinolaryngology, Sultan Abdulhamid Han Training and Research Hospital, Istanbul, Turkey \\ ${ }^{3}$ Department of Otorhinolaryngology, Tepecik Training and Research Hospital, Istanbul, Turkey
}

\begin{abstract}
OBJECTIVE: Laryngeal squamous cell carcinoma (LSCC) is the most frequently seen head and neck malignancy. Despite improvements in the treatment modalities within the last 20 years, the desired improvement in survival outcomes has not been achieved yet which led researchers to investigate factors that might affect prognosis in LSCCs.

METHODS: A total of 116 previously operated patients were included in this study. To assess systemic inflammation, neutrophil/lymphocyte ratio (NLR) and platelet/lymphocyte ratio (PLR) were calculated. The cut-off values for NLR and PLR were accepted as 2.79 and 112, respectively. To evaluate intratumoral inflammation, hematoxylin-eosin stained tumor sections were evaluated. Tumor-infiltrating lymphocyte (TIL) densities in the tumor area were scored as $1+, 2+$ and $3+$.

RESULTS: The mean overall survival was $29.5 \pm 17.7$ months. In univariate analysis, a statistically significant correlation was seen between age group of 60 years, tumor stage, site and $O S(p=0.025, p=0.026, p=0.029)$. There was no statistically significant relationship between PLR, NLR and TIL density and OS. In the multivariate analysis, the 60-year-old group and tumour stage were still significantly associated with the OS $(p=0.033, p=0.046)$.

CONCLUSION: Age and tumor stage were significantly associated with survival in our study, but contrary to the literature, no correlation was found between local and systemic inflammatory response.

Keywords: Inflammation; laryngeal carcinoma; prognosis.

Cite this article as: Kucuk U, Ekmekci S, Bozkurt P, Bulgurcu S, Cukurova I. Relationship between local and systemic inflammatory response and prognosis in laryngeal squamous cell carcinoma. North Clin Istanb 2020;7(2):180-184.
\end{abstract}

$\mathrm{T}$ he larynx is the most common site of head and neck cancers and laryngeal squamous cell carcinoma (LSCC) is the most frequently seen head and neck malignancy $[1,2]$. Treatment modalities are surgery, radiotherapy, chemotherapy, or a combination of them [2]. Within the last 20 years, improvement in LSCC survival outcomes is minimal despite improvements in treatment modalities [3], which has led researchers to investigate factors that may affect prognosis in LSCCs. There are studies in the literature reporting that neutrophil/lymphocyte ratio (NLR) and platelet/lymphocyte ratio (PLR), which reflect systemic inflammation, are effective predictors associated with decreased survival rates in various types of cancer [4].

However, some studies have indicated that the increased density of tumor-infiltrating lymphocytes (TILs), which demonstrates intratumoral inflammation, is a good prognostic factor [4]. Herein the relationship

Received: March 21, 2019 Accepted: November 09, 2019 Online: December 03, 2019

Correspondence: Dr. Suphi BULGURCU. Sultan Abdulhamid Han Egitim ve Arastirma Hastanesi, Haydarpasa, Istanbul, Turkey.

Tel: +902165422020 e-mail: suphibulg@yahoo.com

(c) Copyright 2020 by Istanbul Provincial Directorate of Health - Available online at www.northclinist.com 
between systemic and local inflammatory response and total survival was investigated in cases with LSCC.

\section{MATERIALS AND METHODS}

Local ethics committee approval was obtained for this study. A total of 116 patients who were operated in our hospital with the diagnosis of LSCC, whose clinical follow -up records, and archival data were available were included in this study. Patients' age, sex, tumor location and tumor size were learnt from pathology reports, while survival data and tumor stages from the otorhinolaryngology clinic. To assess systemic inflammation, whole blood results were routinely obtained from all patients within the preoperative 1 week, and NLR and PLR were calculated by recording blood neutrophil, lymphocyte and platelet ratios.

The cut-off values for NLR and PLR were accepted as 2.79 and 112, respectively [4].

To evaluate intratumoral inflammation, hematoxylineosin stained tumor sections of the cases in our archive were re-evaluated by two pathologists (Olympus BX51, Japan). Tumor sections with the highest TIL densities were identified. TIL densities in the tumor area were scored as $1+(<30 \%), 2+(30-60 \%)$ and $3+(60 \%)$ scans [5] under $100 \mathrm{x}$, and 400x magnification.

\section{Statistical Analyses}

Statistical analyses were performed using the Statistical Package of Social Sciences version 24 (IBM Corp: Armonk, NY, USA). OS rates were estimated using the Kaplan-Meier method. The log-rank test was used to evaluate survival rates in univariate analyzes. The possible factors identified with the univariate analyzes were further entered into the Cox regression analyses, with backward selection, to determine independent predictors of OS. Only those with clinical significance were included. A p-value $<0.05$ was considered to indicate statistical significance.

\section{RESULTS}

The mean age of the study population $(n=116)$ which consisted of five (4.3\%) female, and 111 (95.7\%) male patients was $61 \pm 9.50(\min : 34$, max: 82$)$ years. The patients were either $\leq 60(n=59: 50.9 \%)$ or $>60(n=57$; $49.1 \%)$ years of age. Eighty six $(74.1 \%)$ patients were alive, and $30(25.9 \%)$ patients died. The median OS was
TABLE 1. Clinicopathologic features of the cases NLR, PLR, TIL

\begin{tabular}{lc} 
Characteristics & $\%$ \\
\hline Age (years), mean \pm SD & $61 \pm 9.50$ \\
60 -year-old group $(\leq 60 />60)$ & $50.9 / 49.1$ \\
Gender (male/female) & $95.7 / 4.3$ \\
Survey (alive/died) & $74.1 / 25.9$ \\
Overall survival (month), median \pm SD & $26.5 \pm 17.7$ \\
Tumorsubsite & \\
(supraglottic/glottic/subglottic) & $17.2 / 71.6 / 11.2$ \\
Tumorstages (I/II/III/IV) & $37.1 / 8.6 / 19 / 35.3$ \\
NLR (<2.79/ $\geq 2.79)$ & $40.5 / 59.5$ \\
TLR (<112/ $\geq 112)$ & $17.2 / 82.8$ \\
TIL (mild/moderate/severe) & $27.6 / 37.1 / 35.3$ \\
\hline
\end{tabular}

NLR: Neutrophil to lymphocyte ratio; PLR: Platelet/to lymphocyte ratio; TIL: Tumo $r$ infiltrating lymphocyte; SD: Standard deviation.

26.5 \pm 17.7 months (min: 2 months, max: 81 months).

Twenty (17.2\%) supraglottic, 83 (71.6\%) glottic and $13(11.2 \%)$ subglottic. $43(37.1 \%)$ tumors with stages 1 , $10(8.6 \%)$ stage $2,22(19 \%)$ stage 3 and $41(35.3 \%)$ were detected. Blood NLR was either $<2.79$ in $47(40.5 \%)$ or $\geq 2.79$ in $69(59.5 \%)$ and TLR was $<112$ in 20 (17.2\%), and $\geq 112$ in $96(82.8 \%)$ cases.

In the hematoxylin-eosin stained sections, 32 mild (27.6\%), 43 moderate (37.1\%) and 41 severe (35.3\%) TIL densities were detected in indicated number of cases when the patients with moderate and severe inflammation were accepted as severe, the density of TIL was mild in $32(27.6 \%)$ and severe in $84(72.4 \%)$ patients. The clinicopathological characteristics are summarized in Table 1 .

The relationship between data and OS were evaluated individually. Accordingly, statistically significant relationship was detected between age groups $(<60$, $\geq 60$ ), tumor stages (stages $1,2,3,4$ ), location and OS in univariate analysis $(\mathrm{p}=0.025, \mathrm{p}=0.026, \mathrm{p}=0.029)$ (Fig. $1)$. There was no statistically significant correlation between PLR, NLR and TIL density and OS $(p=0.745$, $\mathrm{p}=0.987, \mathrm{p}=0.107$ ).

When the data about 60 year- old group, tumor stage, and location, which were statistically significant correlated with $O S$ in univariate analysis, were evaluated using multivariate analysis, a 60-year-old group and tumor stage were still significantly associated with the OS $(p=0.033, p=0.046)$ (Table 2). 

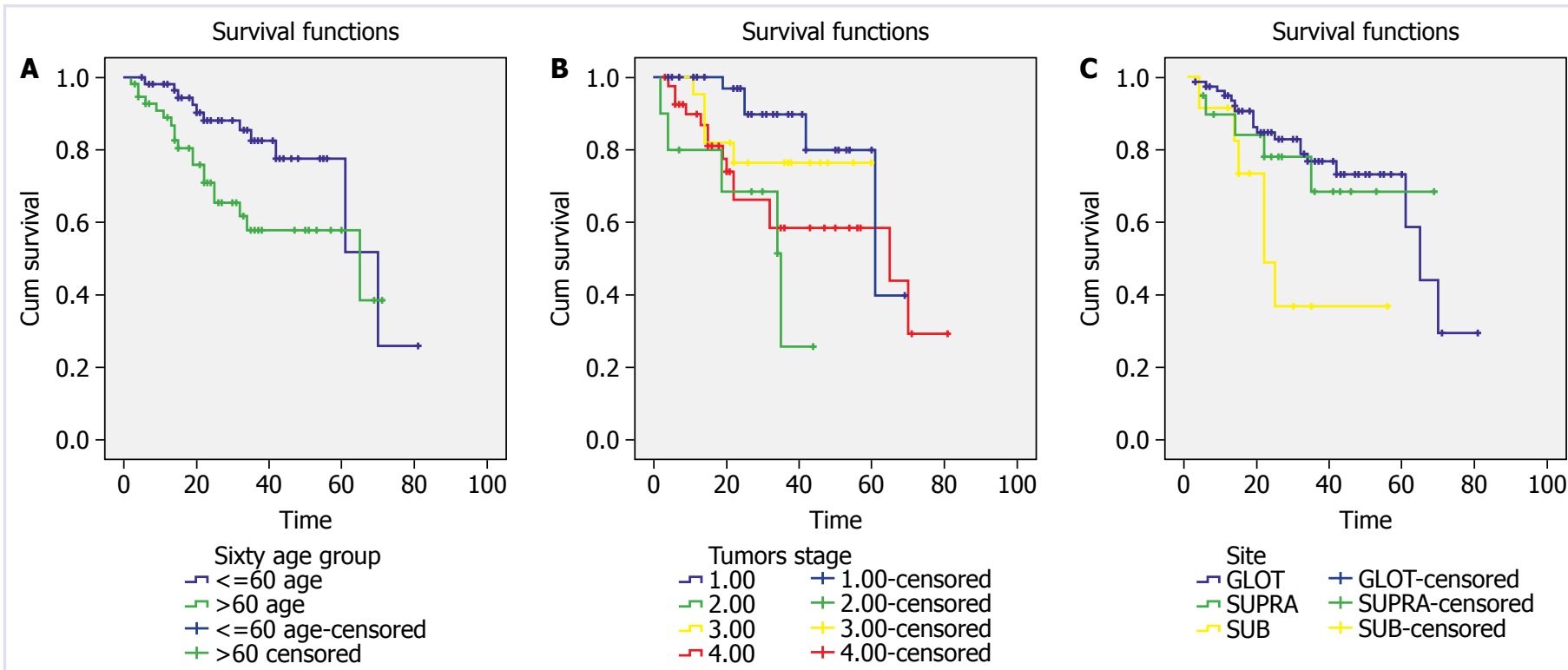

FIGURE 1. (A) Kaplan-Meier curves of overall survival stratified according to the 60-year-old group. (B) Kaplan-Meier curves of Overall Survival stratified according to tumor stages. (C) Kaplan-Meier curves of Overall Survival stratified according to tumor subsite.

TABLE2. Univariate and Multivariate analysis of prognostic factors for overall survival

\begin{tabular}{|c|c|c|c|c|c|c|}
\hline Variables & Total $\mathrm{N}$ & $\mathrm{N}$ of death & $\mathrm{HR}$ & Univariate $\mathrm{p}$-value & $\mathrm{HR}$ & Multivariate $p$-value \\
\hline \multicolumn{7}{|c|}{60 years old group } \\
\hline$\leq 60$ age & 59 & 11 & 11.504 & 0.025 & 2.277 & 0.033 \\
\hline$>60$ age & 57 & 19 & 26.762 & & & \\
\hline \multicolumn{7}{|l|}{ Tumor stages } \\
\hline Stage 1 & 43 & 5 & 3.813 & 0.026 & Reference & \\
\hline Stage 2 & 10 & 5 & 4.924 & & 4.319 & 0.024 \\
\hline Srage 3 & 22 & 5 & 4.153 & & 2.032 & 0.270 \\
\hline Stage 4 & 41 & 15 & 5.494 & & 2.929 & 0.046 \\
\hline \multicolumn{7}{|l|}{ Tumor subsite } \\
\hline Glottic & 83 & 19 & 3.934 & 0.029 & Reference & \\
\hline Supraglottic & 20 & 5 & 6.013 & & 1.240 & 0.686 \\
\hline Subglottic & 13 & 13 & 6.231 & & 2.370 & 0.081 \\
\hline
\end{tabular}

HR: Hazard ratio; Cl: Confidence interval; SD: Standard deviation

\section{DISCUSSION}

LSCC is the most frequent malignancy of the head and neck region and is the major cause of morbidity and mortality [2]. American Cancer Society reported that despite improvements in the treatment modalities, patients with laryngeal cancer had a tendency to decline at 5-year survival rates [6]. This has prompted researchers to search for effective biomarkers that can aid in predicting high- -risk patients with poor prognosis or early recurrence. Recent findings as a result of the studies suggest that the interaction between tumor cells and tumor microenvironment may play effective roles in tumor aggressiveness and treatment resistance [7].

Recently, it has been suggested that inflammation and immune status associated with the tumor are likely to be involved in the development of cancer and in the progression of the disease. This can be explained by the change in 
the distribution of circulating blood cells (such as relative neutrophilia, thrombocytosis and relative lymphopenia) in the cancer-related systemic inflammatory response [4, 8]. Cytokines and chemokines produced by the increased number of neutrophils are thought to be effective in the growth and progression of the tumor. Growth factors secreted by platelets are thought to exert important effects on tumor growth and dissemination due to their roles in increased stimulation of angiogenesis and microvessel permeability [4]. Lymphocytes are known to play an important role in the cancer-related host immune response by suppressing proliferation and metastasis of tumor cells, and this role may be thought to be depressed because of the presence of relative lymphopenia [4].

Recent studies have reported that NLR and PLR are effective markers of systemic host immunity and associated with advanced stage and poor prognosis in various types of solid tumors, in addition to their being are predictive factors for decreased survival $[2,4]$.

Tu et al. reported that high NLR in 141 LSCC series was associated with short disease-free survival (DFS) and short-term OS in univariate and multivariate analysis [2]. They also reported that in univariate analysis OS correlated statistically significantly with stage, location, the histologic grade of the tumor, and NLR; however, in multivariate analysis significant correlations among T classification, lymph node metastasis and NLR were maintained.

Fu et al. indicated that in univariate analysis age group (60 years), alcohol consumption, location, stage, histology grade of the tumor and NLR were significantly associated with cancer-specific survival in a series of 420 cases with LSCC. However, in multivariate analysis, correlations among age, alcohol consumption, lymph node metastasis and NLR were still maintained [8]. Similar findings have been reported in the same study for OS [9].

In univariate analysis, Wang et al. reported that the preoperatively higher PLR and NLR were associated with decreased OS and DFS in their series of 120 cases, but in the multivariate analysis, only higher PLR retained its correlation [4]. In the same study, it was also found that there was no statistically significant relationship between tumor location and tumor stage with OS and RFS.

In our series, univariate analysis showed a statistically significant relationship between age (60 age group), tumor stage and location of the tumor and OS. However, a statistically significant relationship was not observed between NLR, PLR and OS in our series, unlike the literature findings. In our study, in the multivariate analysis 60-year-old group and tumour stage continued to have a significant association with the OS $(p=0.033, p=0.046)$.

Unlike studies reporting that the increased intensity of the systemic inflammatory response is associated with poor prognosis, some studies have indicated that intratumoral inflammatory response, which response to local inflammatory response, is a good prognostic marker [4]. Chronic inflammatory cells infiltrating the tumor consist of lymphocytes, plasma cells, and macrophages [10]. In particular, TIL is thought to reflect host immune responses to the tumor [4]. In studies of the head and neck region SCCs, it has been reported that increased TIL density is associated with prolonged survival $[4,5,11]$. In a series of 120 LSCC cases by Wang et al., TIL density of tumors was morphologically evaluated, and two groups as low TIL and high TIL were determined [4]. In their studies, they detected higher recurrence rates in the low TIL group when compared with the high TIL group, and they reported that low TIL density could be used as a marker for early recurrence [4]. In our study, there was no statistically significant relationship between TIL density and OS, unlike the literature data.

However, in studies that determined TIL subtypes, it was reported that lymphocytes characterized as CD8positive T-cytotoxic/suppressor cells were correlated with prognosis, whereas FoxP3 cells, a subset of CD4positive $T$ helper lymphocytes, did not exert a negative effect on survival [11]. The differences in TIL assessment methods in studies suggest that the lack of a generally accepted standardized cut-off value may explain our outcome different from that of the literature.

\section{Conclusion}

In recent studies, the relationship of host immunity status to tumor recurrence, response to treatment and survival has begun to be better understood, and its prognostic significance has been reported in a wide variety of tumors, including head and neck region SCCs. However, in our study of LSCC cases, a significant correlation was observed between especially age and tumor stage with survival contrary to the literature data, a significant correlation between local and systemic inflammatory response and survival was not detected which suggested lack of a standardized method of assessing the local inflammatory response and heterogeneity in the treatment modalities. 
Ethics Committee Approval: The University Of Health Sciences Izmir Tepecik Health Practice Research Center Non-interventional Ethics Committee provided the ethics committee approval for this study (date: 25/10/2018, number: 2018/ 12-4).

Conflict of Interest: No conflict of interest was declared by the authors.

Financial Disclosure: The authors have declared that they didn't receive any financial support of this study.

Authorship Contributions: Concept - UK, SE; Design - UK, SE, PB; Supervision - IC, SB, PB; Fundings - UK; Materials - UK, SB; Data collection and/or processing - SB, IC; Analysis and/or interpretation - SE, SB; Literature review - UK, IC; Writing - UK, SB; Critical review - PB, SE.

\section{REFERENCES}

1. Santos TS, Estêvão R, Antunes L, Certal V, Silva JC, Monteiro E. Clinical and histopathological prognostic factors in locoregional advanced laryngeal cancer. J Laryngol Otol 2016;130:948-53. [CrossRef]

2. Tu XP, Qiu QH, Chen LS, Luo XN, Lu ZM, Zhang SY, et al. Preoperative neutrophil-to-lymphocyte ratio is an independent prognostic marker in patients with laryngeal squamous cell carcinoma. BMC Cancer 201520;15:743.[CrossRef]

3. Yilmaz M, Karatas OF, Yuceturk B, Dag H, Yener M, Ozen M. AlphaB-crystallin expression in human laryngeal squamous cell carcinoma tissues. Head Neck 2015;37:1344-8. [CrossRef]

4. Wang J, Wang S, Song X, Zeng W, Wang S, Chen F, et al. The prog- nostic value of systemic and local inflammation in patients with laryngeal squamous cell carcinoma. Onco Targets Ther 2016;9:7177-85. [CrossRef]

5. Siegel R, Ma J, Zou Z, Jemal A. Cancer statistics, 2014. CA Cancer J Clin 2014;64:9-29. [CrossRef]

6. de Ruiter EJ, Ooft ML, Devriese LA, Willems SM. The prognostic role of tumor infiltrating T-lymphocytes in squamous cell carcinoma of the head and neck: A systematic review and meta-analysis. Oncoimmunology 2017;6:e1356148. [CrossRef]

7. Rassouli A, Saliba J, Castano R, Hier M, Zeitouni AG. Systemic inflammatory markers as independent prognosticators of head and neck squamous cell carcinoma. Head Neck 2015;37:103-10. [CrossRef]

8. Fu Y, Liu W, OuYang D, Yang A, Zhang Q. Preoperative Neutrophilto-lymphocyte Ratio Predicts Long-term Survival in Patients Undergoing Total Laryngectomy With Advanced Laryngeal Squamous Cell Carcinoma: A Single-center Retrospective Study. Medicine (Baltimore) 2016;95:e2689. [CrossRef]

9. Turner N, Wong HL, Templeton A, Tripathy S, Whiti Rogers T, Croxford $\mathrm{M}$, et al. Analysis of local chronic inflammatory cell infiltrate combined with systemic inflammation improves prognostication in stage II colon cancer independent of standard clinicopathologic criteria. Int J Cancer 2016;138:671-8. [CrossRef]

10. Nguyen N, Bellile E, Thomas D, McHugh J, Rozek L, Virani S, et al; Head and Neck SPORE Program Investigators. Tumor infiltrating lymphocytes and survival in patients with head and neck squamous cell carcinoma. Head Neck 2016;38:1074-84. [CrossRef]

11. Vassilakopoulou M, Avgeris M, Velcheti V, Kotoula V, Rampias T, Chatzopoulos K, et al. Evaluation of PD-L1 Expression and Associated Tumor-Infiltrating Lymphocytes in Laryngeal Squamous Cell Carcinoma. Clin Cancer Res 2016;22:704-13. [CrossRef] 